\title{
Frequency of Loud Snoring and Metabolic Syndrome among Korean Adults: Results from the Health Examinees (HEXA) Study
}

\author{
Claire E. Kim ${ }^{1,2}$ (D), Sangah Shin ${ }^{3}$, Hwi-Won Lee ${ }^{1,2}$, Jiyeon Lim ${ }^{1}$, Jong-Koo Lee ${ }^{4,5}$ \\ and Daehee Kang 1,2,6,* \\ 1 Department of Preventive Medicine, College of Medicine, Seoul National University, 103 Daehakro, \\ Jongnogu, Seoul 03080, Korea; claireekim@snu.ac.kr (C.E.K.); hwiwon@snu.ac.kr (H.-W.L.); \\ jiyeonlim@snu.ac.kr (J.L.) \\ 2 Department of Biomedical Sciences, College of Medicine, Seoul National University, 103 Daehakro, \\ Jongnogu, Seoul 03080, Korea \\ 3 Department of Food and Nutrition, Chung-Ang University, Gyeonggi-do 17546, Korea; ssa8320@snu.ac.kr \\ 4 JW Lee Center for Global Medicine, College of Medicine, Seoul National University, \\ IhwaJang-gil 71 Jongno-gu, Seoul 03087, Korea; docmohw@snu.ac.kr \\ 5 Department of Family Medicine, Seoul National University Hospital, 101 Daehakro, Jongnogu, \\ Seoul 03080, Korea \\ 6 Institute of Environmental Medicine, Seoul National University Medical Research Center, 103 Daehakro, \\ Jongnogu, Seoul 03080, Korea \\ * Correspondence: dhkang@snu.ac.kr; Tel.: +82-2-740-8396
}

Received: 14 September 2017; Accepted: 22 October 2017; Published: 26 October 2017

\begin{abstract}
Studies regarding the association between snoring and metabolic abnormalities have been inconsistent. We examine whether snoring frequency and obstructive sleep apnea markers are associated with metabolic syndrome (MetS) among Koreans aged 40-69 years. A total of 72,885 subjects ( 24,856 men, 48,029 women) from the Health Examinees Gem study between 2009 and 2013 were included. Snoring frequency was grouped into five categories (never, $1-3 /$ month, 1-3/week, 4-5/week, 6+/week). Obstructive sleep apnea markers included breathing interruptions and awakenings. Adjusted odds ratios (ORs) and $95 \%$ confidence intervals (95\% CIs) were calculated through logistic regression. Compared with non-snorers, those who snore $6+/$ week were associated with increased odds for MetS (OR: 2.07, 95\% CI: 1.91-2.25, p-trend < 0.0001 among men; OR: 1.45, CI: 1.33-1.58, $p$-trend $<0.0001$ among women). Snoring frequency is associated with MetS and its components in both men and women. Snoring and obstructive sleep apnea markers are important indicators of sleep quality, which may facilitate early detection of sleep disorders and further complications such as MetS.
\end{abstract}

Keywords: snoring; metabolic syndrome; epidemiology; health examinees study; Korean

\section{Introduction}

Snoring, a physical phenomenon caused by high-frequency vibrations of the soft palate, pharyngeal walls, the tongue and the epiglottis partially blocking the upper airway, has been increasingly regarded as a pathophysiologic entity that poses risk for adverse health effects [1]. Specifically, it has been identified as an important indicator of sleep quality and consequentially as a potential marker for sleep disorders such as obstructive sleep apnea (OSA) [2,3]. Accordingly, validated tools measuring OSA, such as the Basic Nordic Sleep Questionnaire, pose several questions that aim to assess "habitual snoring" [4,5].

Despite the growing number of snoring studies, methodological differences such as the categorization of snoring frequency and varying OSA-related questions have produced mixed results. Studies conducted 
in Western and Asian countries on snoring have tended to dichotomize the exposure to be the presence of "habitual" snoring with no consensus on the number of snoring accounts per week [6,7]. Furthermore, few studies assessed the effects of breathing interruptions and awakenings, both of which are common symptoms of OSA $[4,8,9]$. Examination of dose-response snoring frequency with finer categories as well as the other OSA markers may improve clinical interviews and epidemiological questionnaires in predicting sleep-related health outcomes. Moreover, self-report questionnaires may be a low-cost, noninvasive tool for large epidemiological studies to screen the general population for people who may be unknowingly at risk for OSA and its subsequent health outcomes [5,10,11].

Metabolic syndrome (MetS) is characterized by a cluster of cardiovascular risk factors such as elevated waist circumference, high triglyceride levels, low high-density cholesterol levels, hypertension, and high fasting glucose. Previous observational studies suggest that snoring is associated with MetS and its components [12-15] including dyslipidemia [16], hypertension [17], and diabetes [6]. Additionally, prospective studies have identified snoring as an independent risk factor for MetS [18]. Given the high prevalence of MetS among Korean adults [19], identifying modifiable risk factors such as snoring is warranted. Additionally, the prevalence of snoring and OSA among the Korean population is similar to those of Western societies, despite the overall lower average BMI among Koreans. Therefore, studying the relationship between snoring and MetS among Koreans may provide insight into the non-obesity related risk factors contributing to the pathogenesis of MetS [20].

The current study aimed to examine snoring frequency, breathing interruptions, and awakenings with the odds for MetS and its components. To consider the gender differences in the prevalence of snoring [7], we explored whether the studied relationships differed between men and women. We also performed a subgroup analysis by normal and overweight BMI to address potential confounding by BMI. To our knowledge, this is the largest study assessing the gender stratified, dose-response relationship of snoring frequency and OSA markers with MetS and its components.

\section{Materials and Methods}

\subsection{Study Population}

The Health Examinees (HEXA) study is a large-scale community-based genomic survey conducted in Korea 2004-2013 of adults aged 40-69. Description of the HEXA study rationale, study design, and baseline characteristics can be found in its introductory papers [21,22]. Updated from the previously published HEXA studies, the current study uses the HEXA Gem (HEXA-G) participant sample which includes additional eligibility criteria on the participating sites (i.e., health examination centers and training hospitals) and is outlined in detail in previous literature [23]. In this study, HEXA-G data from years 2009-2013 were used as prior surveys did not include snoring-related questions. Of the eligible HEXA-G ( $n=74,257)$ study subjects, we excluded those without information on any of the MetS components $(n=170)$ : snoring (Yes/No) $(n=491)$ or snoring frequency $(n=711)$. The final analytic sample included a total of 72,885 subjects with 24,856 men and 48,029 women (Figure 1 ). The HEXA study was approved by the Ethics Committee of the Korean Health and the institutional review boards of all participating hospitals (IRB No. E-1503-103-657). All study participants provided informed consent prior to entering the study. 


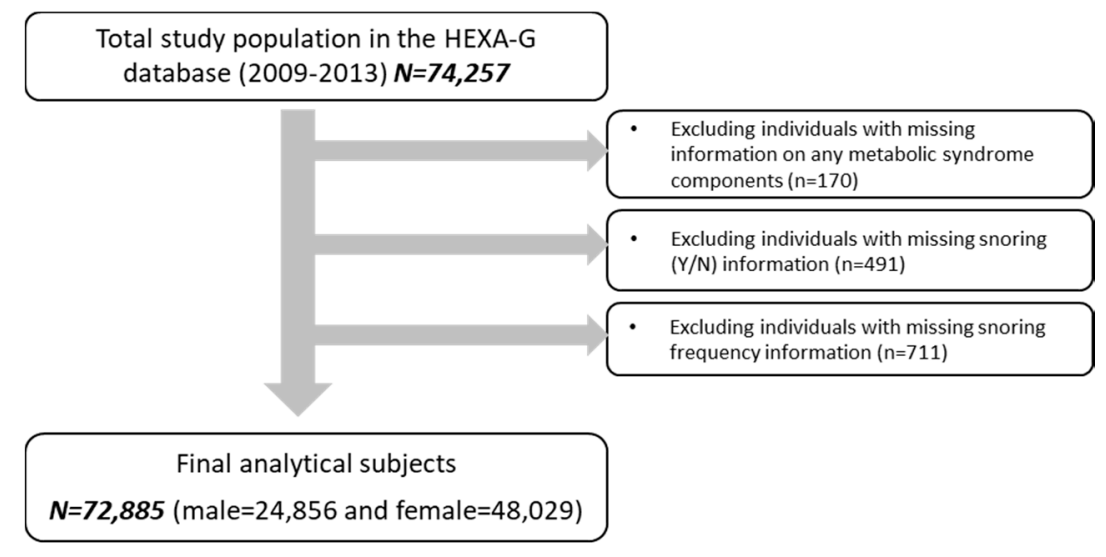

Figure 1. Flow diagram of analytical sample, the HEXA Gem (HEXA-G) 2009-2013.

\subsection{Assessment of Snoring Frequency and Symptoms}

Presence of snoring was assessed with the following question: "I have been told that I snore loudly" with a Yes or No response. For those who answered "Yes", a follow-up question regarding snoring frequency was asked: "How often do you snore?" with the available five category responses: very rarely, 1-3/month, 1-3/week, 4-5/week, 6+/week. In the current study, participants who answered, "No" to the presence of snoring and "Very rarely" to the snoring frequency question were grouped into the "Never" category and served as the reference group for the final analysis.

Breathing interruptions were assessed with a Yes or No question: "I have been told (by someone who shares the room with me) that I've displayed breathing interruptions while snoring". Awakenings were assessed with a Yes or No question: "There were times in which I have woken up from snoring". In the final analysis, for both snoring symptoms, the "No" category, which includes both non-snorers and snorers without the OSA markers, was set as the reference group. This reference group allowed the evaluation of each OSA marker "independently" in its association with MetS and its components. As these markers are highly correlated, snoring frequency and presence of awakenings (for breathing interruptions, vice versa) were not adjusted in the final model.

\subsection{Definition of Metabolic Syndrome (MetS)}

The current study defined MetS using the National Cholesterol Education Program Adult Treatment Panel III [24]. Participants who met three or more of the following criteria were classified as having MetS: (1) waist circumference (WC) $\geq 90$ and $\geq 80 \mathrm{~cm}$ for men and women, respectively; (2) triglycerides (TG) $\geq 150 \mathrm{mg} / \mathrm{dL}$ or drug treatment for elevated triglycerides; (3) high-density lipoprotein cholesterol (HDL-C) $\leq 40$ and $\leq 50 \mathrm{mg} / \mathrm{dL}$ in men and women, respectively; (4) systolic blood pressure $(\mathrm{BP}) \geq 130$, diastolic $\mathrm{BP} \geq 85 \mathrm{mmHg}$ or drug treatment for elevated BP; and (5) fasting glucose $\geq 100 \mathrm{mg} / \mathrm{dL}$ or drug treatment for elevated fasting glucose.

\subsection{Covariates}

Sociodemographic factors such as age (continuous), BMI (continuous), education, occupation, marital status, and menopausal status (women only) were assessed. Education was categorized as middle school or below, high school graduate, and college or above. Occupation was categorized as non-manual, manual, and unemployed. Marital status was categorized into married or single (including never married, separated, divorced, or bereaved). Menopausal status was classified as either post-menopausal women who have gone a year without menstrual flow or pre-menopausal women who currently experience monthly menstrual cycles.

Other covariates included lifestyle factors such as sleep duration, smoking, alcohol drinking, and regular exercise. Sleep duration represented the average amount of sleep per day, a continuous 
variable measured in minutes. Smoking status was ascertained by posing the following question: "Have you smoked more than 5 packs of cigarettes (100 cigarettes) in your lifetime?" People who responded as never having smoked 100 cigarettes were defined as non-smokers; subjects who had smoked $\geq 100$ cigarettes during their lifetime but did not smoke at the time of the survey were classified as past smokers; subjects who had smoked $\geq 100$ cigarettes during their lifetime and still smoked cigarettes at the time of the survey were classified as current smokers. Alcohol drinking status was determined by the following question: "Are you unable to consume alcohol or do you refuse to do so (for religious reasons, etc.)?" Respondents who answered "yes" were determined as non-drinkers. Drinkers who have answered the question, "Do you still drink" with "no" were also categorized as non-drinkers and with "yes" as current drinkers. Exercise was assessed by the question: "Do you participate in any sport regularly (enough to sweat)? Respondents who answered "no" were assigned to the non-regular exercise group; subjects who responded "yes" were assigned to the regular exercise group. For all covariates, missing data was categorized as "unknown".

\subsection{Statistical Analysis}

All estimates were calculated separately by gender to investigate the gender differences in the association between snoring frequency and symptoms and MetS. Gender interaction $p$-values were assessed by the likelihood ratio tests with the use of a cross-product term. A chi-square test (for categorical variables) and ANOVA (for continuous variables) were performed to analyze the basic characteristics of the study population with respect to snoring categories. All variables were tested for normality using the skewness-kurtosis test. For the main OR analyses, an adjusted model including age (continuous), BMI (continuous; not adjusted for WC OR), education ( $\leq$ middle school, high school, $\geq$ college, unknown), occupation (manual, non-manual, unemployed, unknown), marital status (married, single, unknown), menopausal status (pre-, post-, unknown; women only), smoking (never, past, current, unknown; men only), alcohol drinking (current, non, unknown), regular exercise (yes, no unknown), and sleep duration (continuous) as covariates was used. Smoking was excluded from the model for women because the prevalence of smoking $(\sim 6 \%)$ was very low among all snoring categories. As BMI is a modifier of the association between snoring and MetS, BMI was adjusted in the final model. However, since BMI is highly correlated with waist circumference, the final model excluded BMI from its WC OR calculation. Moreover, to account for comorbidities, a sensitivity analysis by excluding subjects with a history of type 2 diabetes, hypertension, and dyslipidemia was performed. Supplementary analyses involving BMI subgroups and number of OSA markers (i.e., snoring, breathing interruptions, awakenings) on the odds for MetS were also conducted.

Multivariable logistic regression models were used to calculate prevalence odds ratios (ORs) and $95 \%$ confidence intervals (95\% CIs). We tested the linear trend across snoring frequency using general linear regression. All of the $p$-values were two-sided, and statistical significance was set at below 0.05. All statistical analyses were conducted using the SAS software version 9.4 (SAS Institute, Cary, NC, USA).

\section{Results}

Baseline characteristics of the study population by snoring frequency are summarized in Table 1. About $14.6 \%$ of men and $7.3 \%$ of women indicated snoring $6+/$ week. All selected covariates for the final analysis, with the exception of occupation in men and alcohol drinking in women, differed with statistical significance across the snoring frequency categories.

The overall prevalence of MetS was $29.3 \%$ in men and $24.9 \%$ in women (Table 2). There were significant differences in the prevalence of MetS and all five of its components based on snoring frequency in both men and women (MetS and all five components $p<0.0001)$. The odds ratios (ORs) and $95 \%$ confidence interval $(95 \%$ CIs) for MetS and its components with snoring categories are presented in Table 3. Using the "never" snoring frequency category as a reference, snoring 6+/week was associated with statistically significant increased odds for MetS in both men and women (OR: 2.07, 
95\% CI: 1.91-2.25, $p$-trend $<0.0001$ among men; OR: 1.45, 95\% CI: 1.33-1.58, $p$-trend $<0.0001$ among women) when adjusted for age, BMI, education, occupation, marital status, smoking (men only), alcohol drinking, regular exercise, menopausal status (women only), and sleep duration.

Table 1. Baseline characteristics ${ }^{a}$ by snoring frequency, the Health Examinees Gem (HEXA-G) 2009-2013.

\begin{tabular}{|c|c|c|c|c|c|c|c|}
\hline & \multicolumn{7}{|c|}{ Snoring Frequency } \\
\hline Men, $n$ & 24856 & $9555(38.4)$ & 4857 (19.5) & $5190(20.9)$ & $1634(6.6)$ & $3620(14.6)$ & \\
\hline Age, years & $53.8 \pm 8.5$ & $53.6 \pm 8.8$ & $53.7 \pm 8.4$ & $53.5 \pm 8.2$ & $54.9 \pm 8.1$ & $53.9 \pm 8.4$ & $<0.0001$ \\
\hline BMI, $\mathrm{kg} / \mathrm{m}^{2}$ & $24.4 \pm 2.8$ & $23.7 \pm 2.6$ & $24.4 \pm 2.6$ & $24.8 \pm 2.7$ & $25.1 \pm 2.8$ & $25.5 \pm 2.9$ & $<0.0001$ \\
\hline Current smokers & $18642(75.0)$ & $6916(72.4)$ & $3573(73.6)$ & 4069 (78.4) & $1274(78.0)$ & $2810(77.6)$ & $<0.0001$ \\
\hline College or above & $9316(37.5)$ & $3540(37.1)$ & $1878(38.7)$ & $1999(38.5)$ & $572(35.0)$ & $1327(36.7)$ & 0.0276 \\
\hline Manual occupation & $11,964(48.1)$ & $4571(47.8)$ & $2360(48.6)$ & $2473(47.7)$ & $777(47.6)$ & $1783(49.3)$ & 0.0701 \\
\hline Married & $23,180(93.3)$ & $8686(90.9)$ & $4585(94.4)$ & $4934(95.1)$ & $1551(94.9)$ & $3424(94.6)$ & $<0.0001$ \\
\hline Sleep duration, hours & $6.9 \pm 1.2$ & $6.9 \pm 1.2$ & $6.9 \pm 1.1$ & $6.9 \pm 1.1$ & $6.9 \pm 1.1$ & $6.9 \pm 1.2$ & 0.0091 \\
\hline Women, $n$ & 48,029 & $25,590(53.3)$ & $10,420(21.7)$ & $6736(14.0)$ & $1779(3.7)$ & $3504(7.3)$ & \\
\hline Regular exercisers & $24,826(51.7)$ & $13,026(50.9)$ & $5691(54.6)$ & 3489 (51.8) & $886(49.8)$ & $1734(49.5)$ & $<0.0001$ \\
\hline College or above & $10,065(21.0)$ & $6197(24.2)$ & $2031(19.5)$ & $1109(16.5)$ & $267(15.0)$ & $461(13.2)$ & $<0.0001$ \\
\hline Manual occupation & $13,046(27.2)$ & $6706(26.2)$ & $2787(26.8)$ & $1948(28.9)$ & $533(30.0)$ & $1072(30.6)$ & $<0.0001$ \\
\hline Married & $41,688(86.8)$ & $22,112(86.4)$ & $9205(88.3)$ & $5861(87.0)$ & $1539(86.5)$ & $2971(84.8)$ & $<0.0001$ \\
\hline Post-menopausal & $28,239(58.8)$ & $13,504(52.8)$ & $6455(62.0)$ & $4551(67.6)$ & $1243(69.9)$ & $2486(71.0)$ & $<0.0001$ \\
\hline Sleep duration, hours & $6.9 \pm 1.2$ & $6.9 \pm 1.2$ & $6.9 \pm 1.2$ & $6.9 \pm 1.2$ & $6.9 \pm 1.2$ & $6.9 \pm 1.3$ & 0.0079 \\
\hline
\end{tabular}

${ }^{\mathrm{a}}$ Values are $n(\%)$ or mean $\pm \mathrm{SD} .{ }^{\mathrm{b}} p$-values for differences among snoring categories were calculated by chi-square tests for categorical variables and ANOVA for continuous variables.

Table 2. Metabolic Syndrome (MetS) ${ }^{a}$ prevalent cases ${ }^{b}$ by snoring frequency, the Health Examinees Gem (HEXA-G) 2009-2013.

\begin{tabular}{|c|c|c|c|c|c|c|c|}
\hline & \multicolumn{7}{|c|}{ Snoring Frequency } \\
\hline & Total & Never & 1-3/month & 1-3/week & 4-5/week & 6+/week & $p$-Value ${ }^{c}$ \\
\hline Men, $n$ & 24,856 & 9555 & 4857 & 5190 & 1634 & 3620 & \\
\hline MetS & $7276(29.3)$ & $2216(23.2)$ & $1355(27.9)$ & 1709 (32.9) & $598(36.6)$ & $1398(38.6)$ & $<0.0001$ \\
\hline $\mathrm{WC} \geq 90 \mathrm{~cm}$ & $6911(27.8)$ & $1990(20.8)$ & $1305(26.9)$ & $1603(30.9)$ & $546(33.4)$ & $1467(40.5)$ & $<0.0001$ \\
\hline Serum TG $\geq 150 \mathrm{mg} / \mathrm{dL}$ & $10,077(40.5)$ & $3428(35.9)$ & $1923(39.6)$ & $2299(44.3)$ & $729(44.6)$ & $1698(46.9)$ & $<0.0001$ \\
\hline Serum HDL-C $\leq 40 \mathrm{mg} / \mathrm{dL}$ & $5793(23.3)$ & $2011(21.1)$ & $1101(22.7)$ & $1207(23.3)$ & $429(26.3)$ & $1045(28.9)$ & $<0.0001$ \\
\hline $\mathrm{BP} \geq 130 / 85 \mathrm{mmHg}$ & $13,323(53.6)$ & $4613(48.3)$ & $2582(53.2)$ & $2982(57.5)$ & $990(60.6)$ & $2156(59.6)$ & $<0.0001$ \\
\hline Fasting glucose $\geq 100 \mathrm{mg} / \mathrm{dL}$ & $8517(34.3)$ & $2998(31.4)$ & $1648(33.9)$ & $1879(36.2)$ & $637(39.0)$ & $1355(37.4)$ & $<0.0001$ \\
\hline Women, $n$ & 48,029 & 25,590 & 104,20 & 6736 & 1779 & 3504 & \\
\hline MetS & $11,980(24.9)$ & $4874(19.1)$ & $2796(26.8)$ & $2205(32.7)$ & $657(36.9)$ & $1448(41.3)$ & $<0.0001$ \\
\hline $\mathrm{WC} \geq 80 \mathrm{~cm}$ & $19,121(39.8)$ & $8172(31.9)$ & $4538(43.6)$ & $3313(49.2)$ & $934(52.5)$ & $2164(61.8)$ & $<0.0001$ \\
\hline Serum TG $\geq 150 \mathrm{mg} / \mathrm{dL}$ & $11,730(24.4)$ & $5288(20.7)$ & $2656(25.5)$ & $2020(30.0)$ & $567(31.9)$ & $1199(34.2)$ & $<0.0001$ \\
\hline Serum HDL-C $\leq 50 \mathrm{mg} / \mathrm{dL}$ & $17,047(35.5)$ & $8206(32.1)$ & $3872(37.2)$ & $2637(39.2)$ & $740(41.6)$ & $1592(45.4)$ & $<0.0001$ \\
\hline $\mathrm{BP} \geq 130 / 85 \mathrm{mmHg}$ & $18,380(38.3)$ & $8249(32.2)$ & $4280(41.1)$ & $3148(46.7)$ & $882(49.6)$ & $1821(52.0)$ & $<0.0001$ \\
\hline Fasting glucose $\geq 100 \mathrm{mg} / \mathrm{dL}$ & $9330(19.4)$ & $4127(16.1)$ & $2128(20.4)$ & $1602(23.8)$ & $474(26.6)$ & $999(28.5)$ & $<0.0001$ \\
\hline
\end{tabular}

a MetS: the presence of 3 or more of the following components: (1) elevated waist circumference (WC); (2) high triglyceride (TG) levels; (3) low high density lipoprotein-cholesterol (HDL-C) level or taking anticholesterol medication; (4) high blood pressure (BP) or taking antihypertensive medicine; (5) high fasting glucose levels or taking medication to treat diabetes mellitus. ${ }^{\mathrm{b}}$ Values are $n(\%) .{ }^{\mathrm{c}} p$-values for differences among snoring categories were calculated by chi-square tests. 
Table 3. Odds ratio (ORs) ${ }^{a}$ of metabolic syndrome (MetS) ${ }^{b}$ by snoring frequency, the Health Examinees Gem (HEXA-G) 2009-2013.

\begin{tabular}{|c|c|c|c|c|c|c|}
\hline & \multicolumn{6}{|c|}{ Snoring Frequency } \\
\hline & Never & 1-3/month & 1-3/week & 4-5/week & $6+/$ week & $p$-Trend \\
\hline Men $n=24,856^{d}$ & 9555 & 4857 & 5190 & 1634 & 3620 & \\
\hline MetS & Ref. & $1.29(1.19-1.40)$ & $1.63(1.51-1.75)$ & $1.85(1.65-2.07)$ & $2.07(1.91-2.25)$ & $<0.0001$ \\
\hline $\mathrm{WC} \geq 90 \mathrm{~cm}$ & Ref. & $1.40(1.29-1.52)$ & $1.69(1.57-1.83)$ & $1.86(1.66-2.09)$ & $2.57(2.37-2.79)$ & $<0.0001$ \\
\hline Serum TG $\geq 150 \mathrm{mg} / \mathrm{dL}$ & Ref. & $1.18(1.09-1.26)$ & $1.39(1.30-1.49)$ & $1.44(1.29-1.60)$ & $1.56(1.45-1.69)$ & $<0.0001$ \\
\hline Serum HDL-C $\leq 40 \mathrm{mg} / \mathrm{dL}$ & Ref. & $1.04(0.95-1.13)$ & $1.04(0.96-1.13)$ & $1.12(0.99-1.27)$ & $1.22(1.11-1.34)$ & $<0.0001$ \\
\hline $\mathrm{BP} \geq 130 / 85 \mathrm{mmHg}$ & Ref. & $1.21(1.13-1.30)$ & $1.44(1.35-1.55)$ & $1.57(1.41-1.75)$ & $1.57(1.45-1.70)$ & $<0.0001$ \\
\hline Fasting glucose $\geq 100 \mathrm{mg} / \mathrm{dL}$ & Ref. & $1.12(1.04-1.21)$ & $1.23(1.14-1.32)$ & $1.32(1.19-1.48)$ & $1.29(1.19-1.40)$ & $<0.0001$ \\
\hline Women $n=48,029^{d}$ & 25,590 & 10,420 & 6736 & 1779 & 3504 & \\
\hline MetS & Ref. & $1.22(1.15-1.29)$ & $1.34(1.25-1.44)$ & $1.40(1.25-1.57)$ & $1.45(1.33-1.58)$ & $<0.0001$ \\
\hline$W C \geq 80 \mathrm{~cm}$ & Ref. & $1.55(1.48-1.63)$ & $1.81(1.71-1.92)$ & $1.98(1.79-2.19)$ & $2.87(2.66-3.09)$ & $<0.0001$ \\
\hline Serum TG $\geq 150 \mathrm{mg} / \mathrm{dL}$ & Ref. & $1.12(1.06-1.18)$ & $1.25(1.17-1.33)$ & $1.25(1.12-1.39)$ & $1.27(1.17-1.38)$ & $<0.0001$ \\
\hline Serum HDL-C $\leq 50 \mathrm{mg} / \mathrm{dL}$ & Ref. & $1.10(1.05-1.16)$ & $1.09(1.02-1.15)$ & $1.11(1.00-1.23)$ & $1.21(1.12-1.30)$ & $<0.0001$ \\
\hline $\mathrm{BP} \geq 130 / 85 \mathrm{mmHg}$ & Ref. & $1.21(1.15-1.27)$ & $1.31(1.24-1.39)$ & $1.34(1.20-1.48)$ & $1.32(1.22-1.43)$ & $<0.0001$ \\
\hline Fasting glucose $\geq 100 \mathrm{mg} / \mathrm{dL}$ & Ref. & $1.13(1.07-1.21)$ & $1.23(1.14-1.31)$ & $1.32(1.18-1.48)$ & $1.33(1.22-1.45)$ & $<0.0001$ \\
\hline
\end{tabular}

a ORs adjusted for: age (continuous), BMI (continuous), education ( $\leq$ middle school, high school, $\geq$ college, unknown), occupation (manual, non-manual, unemployed, unknown), marital status (married, single, unknown), smoking (current, past, never, unknown; men only), alcohol drinking (current, non-, unknown), regular exercise (yes, no, unknown), menopausal status (pre-, post-, unknown; women only), and sleep duration (continuous). ${ }^{b}$ MetS: the presence of 3 or more of the following components: (1) elevated waist circumference (WC); (2) high triglyceride (TG) levels; (3) low high density lipoprotein-cholesterol (HDL-C) or taking anticholesterol medication; (4) high blood pressure (BP) or taking antihypertensive medicine; (5) high fasting glucose levels or taking medication to treat diabetes mellitus. ${ }^{\mathrm{C}}$ Linear trends across snoring frequency categories were calculated by general linear regression. ${ }^{\mathrm{d}}$ Gender $p$-interaction value $<0.0001$; interaction term was assessed by likelihood ratio tests with the use of a cross-product term

Furthermore, in both men and women, snoring 6+/week was associated with increased adjusted (excluding BMI in WC calculation) ORs of elevated waist circumference, hypertriglyceridemia, low HDL-C, elevated blood pressure, and high fasting glucose. Gender differences were observed in the ORs for MetS and its components (all gender $p$-interaction value $<0.0001$ ).

Excluding subjects with either a history of type 2 diabetes, hypertension, or dyslipidemia did not affect the association of snoring frequency with the odds for MetS (OR: 2.12, 95\% CI: 1.90-2.37, p-trend $<0.0001$ in men and OR: 2.16, 95\% CI: 1.94-2.40, $p$-trend $<0.0001$ in women). Moreover, in the subgroup analysis stratified by BMI group, snoring frequency was significantly associated with increased ORs for MetS in both normal and overweight BMI groups (Table S1; BMI $<25 \mathrm{~kg} / \mathrm{m}^{2}$, OR: $1.26,95 \%$ CI: $1.09-1.45$ and $\geq 25 \mathrm{~kg} / \mathrm{m}^{2}$, OR: $1.59,95 \%$ CI: $1.41-1.78$ among men; BMI $<25 \mathrm{~kg} / \mathrm{m}^{2}$, OR: $1.79,95 \%$ CI: $1.58-2.01$ and $\geq 25 \mathrm{~kg} / \mathrm{m}^{2}$, OR: 1.67, 95\% CI: $1.49-1.87$ among women). As BMI is a modifier of the association between snoring and MetS, BMI was adjusted in the final model (with the exception of OR calculation for WC).

The adjusted ORs and CIs for OSA symptoms, breathing interruptions and awakenings, are listed in Table 4. Among 24,783 men who answered the breathing interruption question, 4062 (16.4\%) experienced breathing interruptions; among 47,927 women, 1596 (3.3\%) experienced breathing interruptions. Among 24,824 men who answered the awakening question, 2999 (12.1\%) experienced awakenings; among 47,991 women, 6226 (13.0\%) experienced awakenings. Breathing interruptions were associated with greater odds of MetS (OR: 1.58, 95\% CI: 1.47-1.70 among men; OR: 1.32, 95\% CI: 1.18-1.49 among women), elevated waist circumference (men only), hypertriglyceridemia, low HDL-C (women only), elevated BP, and high fasting glucose. Awakenings were associated with greater odds of MetS (OR: 1.39, 95\% CI: 1.28-1.50 among men; OR: 1.21, 95\% CI: 1.13-1.29), elevated waist circumference (among men), hypertriglyceridemia, low HDL-C (women only), elevated BP, and high fasting glucose were observed. 
Table 4. Odds ratio (ORs) ${ }^{a}$ of metabolic syndrome (MetS) ${ }^{b}$ by OSA markers, the Health Examinees Gem (HEXA-G) 2009-2013.

\begin{tabular}{lcc}
\hline \multicolumn{3}{c}{ Presence of Breathing Interruptions } \\
\hline & Men $(n=4062)$ & Women $^{c}(n=1596)$ \\
\hline MetS & $1.58(1.47-1.70)$ & $1.32(1.18-1.49)$ \\
WC $\geq 90 \mathrm{~cm}(\mathrm{M}) / \geq 80 \mathrm{~cm}(\mathrm{~W})$ & $1.72(1.60-1.84)$ & $1.06(0.92-1.21)$ \\
Serum TG $\geq 150 \mathrm{mg} / \mathrm{dL}$ & $1.28(1.19-1.37)$ & $1.28(1.15-1.43)$ \\
Serum HDL-C $\leq 40 \mathrm{mg} / \mathrm{dL}(\mathrm{M}) / \leq 50 \mathrm{mg} / \mathrm{dL}(\mathrm{W})$ & $1.07(0.99-1.16)$ & $1.18(1.06-1.31)$ \\
$\mathrm{BP} \geq 130 / 85 \mathrm{mmHg}$ & $1.40(1.31-1.51)$ & $1.30(1.17-1.45)$ \\
Fasting glucose $\geq 100 \mathrm{mg} / \mathrm{dL}$ & $1.22(1.13-1.31)$ & $1.26(1.12-1.41)$ \\
\hline & Presence of Awakenings & \\
\hline & Men $(n=2999)$ & Women ${ }^{c}(n=6226)$ \\
\hline MetS & $1.39(1.28-1.50)$ & $1.21(1.13-1.29)$ \\
WC $\geq 90 \mathrm{~cm}(\mathrm{M}) / \geq 80 \mathrm{~cm}(\mathrm{~W})$ & $1.48(1.37-1.61)$ & $0.99(0.90-1.07)$ \\
Serum TG $\geq 150 \mathrm{mg} / \mathrm{dL}$ & $1.24(1.15-1.34)$ & $1.17(1.10-1.25)$ \\
Serum HDL-C $\leq 40 \mathrm{mg} / \mathrm{dL}(\mathrm{M}) / \leq 50 \mathrm{mg} / \mathrm{dL}(\mathrm{W})$ & $0.99(0.91-1.09)$ & $1.08(1.00-1.15)$ \\
$\mathrm{BP} \geq 130 / 85 \mathrm{mmHg}$ & $1.28(1.18-1.38)$ & $1.19(1.10-1.26)$ \\
Fasting glucose $\geq 100 \mathrm{mg} / \mathrm{dL}$ & $1.16(1.07-1.25)$ & $1.18(1.10-1.26)$ \\
\hline
\end{tabular}

a ORs adjusted for: age (continuous), BMI (continuous), education $(\leq$ middle school, high school, $\geq$ college, unknown), occupation (manual, non-manual, unemployed, unknown), marital status (married, single, unknown), smoking (current, past, never, unknown; men only), alcohol drinking (current, non-, unknown), regular exercise (yes, no, unknown), menopausal status (pre-, post-, unknown; women only), and sleep duration (continuous). ${ }^{b}$ MetS: the presence of 3 or more of the following components: (1) elevated waist circumference (WC); (2) high triglyceride (TG) levels; (3) low high density lipoprotein-cholesterol (HDL-C) or taking anticholesterol medication; (4) high blood pressure (BP) or taking antihypertensive medicine; (5) high fasting glucose levels or taking medication to treat diabetes mellitus. ${ }^{c}$ Gender $p$-interaction value $<0.0001$; interaction term was assessed by likelihood ratio tests with the use of a cross-product term.

In another supplementary analysis (Table S2) examining the association between the number of OSA markers and MetS, greater presence of OSA markers was associate with increased OR for MetS (OR: 2.03, 95\% CI: 1.81-2.27, $p$-trend < 0.0001 among men; OR: 1.48, 95\% CI: $1.28-1.72, p$-trend < 0.0001).

\section{Discussion}

In the current study, snoring frequency was associated with increased odds of MetS and its components (elevated waist circumference, hypertriglyceridemia, reduced HDL-C, elevated BP, and high fasting glucose) among Korean adults. Additionally, breathing interruptions had greater odds of MetS, elevated waist circumference (men only), hypertriglyceridemia, low HDL-C (women only), elevated BP, and high fasting glucose. Awakenings were associated with greater odds of MetS and its components in both men and women, with the exception of low HDL-C in men and elevated waist circumference in women.

Given that the association between snoring and OSA markers on MetS is multifactorial, there are several important covariates to consider. Gender differences may be due to upper airway anatomy and physiology such as pharyngeal collapsibility and central respiratory drive [25]. Another study reported that female sex hormones may be protective against sleep-disordered breathing, as postmenopausal women had a higher risk of sleep-disordered breathing than either premenopausal women or postmenopausal women on hormone replacement therapy [26]. Even adjusting for menopausal status, however, gender differences were observed with men having greater odds of MetS and its components by increasing snoring frequency than in women. Furthermore, while the underlying mechanisms of upper airway narrowing are influenced by multiple factors, studies have reported that obesity increases fat deposits, which may affect the metabolic activity of pharyngeal adipose tissue $[27,28]$. To this regard, we performed a subgroup analysis with the following stratified groups: normal $\left(<25 \mathrm{~kg} / \mathrm{m}^{2}\right)$ and overweight/obese $\left(\geq 25 \mathrm{~kg} / \mathrm{m}^{2}\right)$ BMI. An increasing trend of MetS and its components were observed in both BMI groups (Table S1). To account for the differences in OR 
magnitude, BMI was adjusted in the final analysis. Of note, the prevalence of snoring and OSA in the current population is similar to those of Western societies, despite the overall lower average BMI. This suggests that a non-obesity related risk factor may contribute to the pathogenesis of the disease [20]. Lastly, alcohol has been found to increase the pharynx collapsibility via the genioglossal and palatine tensor muscles [29] and therefore was controlled in the final model.

The clinical significance of snoring without diagnosed OSA (hereafter termed "simple snoring") has been controversial. While snoring is one of the main manifestations of OSA, other markers of OSA such as breathing interruptions and awakenings may be a significant contributor more or less to the subsequent health outcomes. Previous studies assessing snoring on the risk of cardiovascular disease (CVD), coronary heart disease (CHD), and its risk factors have noted the caveat that observed negative effects of snoring may be confounded by the presence of OSA [30]. A study in the US reported that snoring was associated with CVD only when it was paired with daytime sleepiness, a symptom of OSA [31]. Another recent study in Finland suggested that, while OSA and snoring related to sleep-disordered breathing are associated with cardiovascular risk, simple snoring is not [10]. Furthermore, a study in Australia using a home sleep apnea monitoring devise concluded that there is no measure of simple snoring associated with all-cause mortality, incident cardiovascular disease or stroke [32]. In contrast, a US study using a polysomnography concluded that simple snoring is associated with carotid artery atherosclerosis [33]. Another recent US study, using a home sleep test, reported that even after adjusting for variables such as BMI and OSA severity, increased simple snoring was associated with increased all-cause mortality [34]. These varying results suggest that snoring with or without OSA merits attention and highlights the need for objective measurements to more accurately quantify snoring frequency and other OSA markers in order to better understand its association with health consequences [35].

There has been less documentation on the association between snoring and MetS. While the biological mechanisms regarding snoring and its effect on MetS is yet uncertain, there are several plausible explanations for the phenomenon. Snoring and the consequent pauses in breathing have been found to increase the sympathetic nervous system [36] and to increase oxidative stress [37,38]. This, in turn, impairs glucose homeostasis and increases glycogenesis and gluconeogenesis leading to insulin resistance $[39,40]$. Further, a study reported raised arterial pressure and respiratory difficulty, which may potentially be a result of increasing micro-arousals, sleep fragmentation, and disrupted restorative value of sleep [14]. Intermittent hypoxia and arousals caused by snoring may also cause a drop in blood pressure, which leads to an increased sympathetic tone, which is involved in the development pulmonary hypertension [41]. Another study found that snoring was linked to a persistent increase in urinary albumin, a risk factor for diabetes [42]. Others observed elevated hemoglobin levels, which has been studied to be a risk factor for all components of MetS [43,44].

While our results display an association between snoring frequency and MetS, there are limitations to consider. First, this is a cross-sectional study, so causality between snoring and MetS cannot be assessed. Future studies using the total HEXA-G sample on sleep duration and subsequent risk of MetS are warranted to confirm these preliminary findings. Second, the questions used to assess snoring frequency and symptoms were based on self-report questionnaire rather than objective measures via polysomnography, allowing for possible misclassification. Nevertheless, self-reported sleep questionnaires are a useful preventative tool in large epidemiological studies [5]. Third, OSA was not measured, so health effects of simple snoring cannot be determined. Finally, while the current study adjusted for all relevant covariates, there may have been residual confounders that were unmeasured. Regardless of these limitations, this is the largest study examining gender stratified, dose-response snoring frequency as well as other markers of OSA with MetS and its components. Specifically, in Korea, snoring-focused literature has been scarce. The association between snoring and MetS among Koreans, who have relatively low average BMI, highlights the need for greater research on the role of non-obesity factors on MetS development. 


\section{Conclusions}

Taken together, our results suggest that greater snoring frequency, breathing interruptions and awakenings are associated with MetS and its components. In clinical practice, screening of individuals at risk for OSA should focus on not only the presence of snoring but also other markers of OSA that indicate its intensity. Additionally, we underline the need for prospective studies to examine the association between snoring and MetS with more objective measures to establish causality. Assessing snoring and OSA markers comprehensively may improve sleep quality and also allow for early recognition of sleep disorders and accompanying health consequences such as MetS.

Supplementary Materials: The following are available online at www.mdpi.com/1660-4601/14/11/1294/s1, Table S1: Odds ratio (ORs) of metabolic syndrome (MetS) by snoring frequency stratified by BMI, the Health Examinees Gem (HEXA-G) 2009-2013, Table S2: Odds ratio (ORs) of metabolic syndrome (MetS) by number of obstructive sleep apnea (OSA) markers, the Health Examinees-Gem (HEXA-G) 2009-2013.

Acknowledgments: Claire E. Kim received a scholarship from the BK21- plus education program provided by the National Research Foundation of Korea. HEXA study was supported by the National Genome Research Institute, Korea Center for Disease Control and Prevention, and by a grant from Seoul National University Hospital (2017). We would like to thank the participants and all members of the HEXA Study Group.

Author Contributions: Claire E. Kim analyzed the data, drafted, reviewed, and edited the manuscript, and contributed to the discussion; Daehee Kang conducted, designed, and supervised the study, reviewed and edited the manuscript, and contributed to the discussion; Sangah Shin, Hwi-Won Lee, and Jiyeon Lim reviewed and edited the manuscript and contributed to the discussion; Jong-Koo Lee conducted, designed, and supervised the study, and reviewed the manuscript.

Conflicts of Interest: The authors declare no conflict of interest.

\section{References}

1. Pevernagie, D.; Aarts, R.M.; De Meyer, M. The acoustics of snoring. Sleep Med. Rev. 2010, 14, $131-144$. [CrossRef] [PubMed]

2. Bhattacharyya, N. Sleep and Health Implications of Snoring: A Populational Analysis. Laryngoscope 2015, 125, 2413-2416. [CrossRef] [PubMed]

3. Sands, M.; Loucks, E.B.; Lu, B.; Carskadon, M.A.; Sharkey, K.; Stefanick, M.; Ockene, J.; Shah, N.; Hairston, K.G.; Robinson, J.; et al. Self-reported snoring and risk of cardiovascular disease among postmenopausal women (from the women's health initiative). Am. J. Cardiol. 2013, 111, 540-546. [CrossRef] [PubMed]

4. Partinen, M.; Gislason, T. Basic Nordic Sleep Questionnaire (BNSQ): A quantitated measure of subjective sleep complaints. J. Sleep Res. 1995, 4 (Supp. 1), 150-155. [CrossRef]

5. Fedson, A.C.; Pack, A.I.; Gislason, T. Frequently used sleep questionnaires in epidemiological and genetic research for obstructive sleep apnea: A review. Sleep Med. Rev. 2012, 16, 529-537. [CrossRef] [PubMed]

6. Xiong, X.; Zhong, A.; Xu, H.; Wang, C. Association between self-reported habitual snoring and diabetes mellitus: A systemic review and meta-analysis. J. Diabetes Res. 2016, 2016, 1958981. [CrossRef] [PubMed]

7. Chan, C.H.; Wong, B.M.; Tang, J.L.; Ng, D.K. Gender difference in snoring and how it changes with age: Systematic review and meta-regression. Sleep Breath. 2012, 16, 977-986. [CrossRef] [PubMed]

8. Tsara, V.; Amfilochiou, A.; Papagrigorakis, M.J.; Georgopoulos, D.; Liolios, E. Guidelines for diagnosis and treatment of sleep-related breathing disorders in adults and children. Definition and classification of sleep related breathing disorders in adults: Different types and indications for sleep studies (part 1). Hippokratia 2009, 13, 187-191. [PubMed]

9. Mbata, G.C.; Chukwuka, J.C. Obstructive Sleep Apnea Hypopnea Syndrome. Ann. Med. Health Sci. Res. 2012, 2, 74-77. [CrossRef] [PubMed]

10. Niiranen, T.J.; Kronholm, E.; Rissanen, H.; Partinen, M.; Jula, A.M. Self-reported obstructive sleep apnea, simple snoring, and various markers of sleep-disordered breathing as predictors of cardiovascular risk. Sleep Breath. 2016, 20, 589-596. [CrossRef] [PubMed]

11. Donovan, L.M.; Patel, S.R. Making the most of simplified sleep apnea testing. Ann. Intern. Med. 2017, 166, 366-367. [CrossRef] [PubMed] 
12. Shin, M.H.; Kweon, S.S.; Choi, B.Y.; Kim, M.K.; Chun, B.Y.; Shin, D.H.; Lee, Y.H. Self-reported snoring and metabolic syndrome: The Korean multi-rural communities cohort study. Sleep Breath. 2014, 18, 423-430. [CrossRef] [PubMed]

13. Sabanayagam, C.; Zhang, R.; Shankar, A. Markers of sleep-disordered breathing and metabolic syndrome in a multiethnic sample of U.S. adults: Results from the national health and nutrition examination survey 2005-2008. Cardiol. Res. Pract. 2012, 2012. [CrossRef] [PubMed]

14. Leineweber, C.; Kecklund, G.; Akerstedt, T.; Janszky, I.; Orth-Gomer, K. Snoring and the metabolic syndrome in women. Sleep Med. 2003, 4, 531-536. [CrossRef]

15. Huang, J.; Qi, J.; Lin, Q.; Li, S.; Chen, G.; Ding, H.; Zhao, J. Snoring and components of metabolic syndrome in southeastern Chinese adults: A community-based study. Clin. Respir. J. 2017. [CrossRef] [PubMed]

16. Brockmann, P.E.; Damiani, F.; Smith, D.L.; Castet, A.; Nunez, F.; Villarroel, L.; Gozal, D. Metabolic consequences of snoring in adolescents and younger adults: A population study in Chile. Int. J. Obes. 2016, 40, 1510-1514. [CrossRef] [PubMed]

17. Cho, N.; Joo, S.; Kim, J.; Abbott, R.D.; Kim, J.; Kimm, K.; Shin, C. Relation of habitual snoring with components of metabolic syndrome in Korean adults. Diabetes Res. Clin. Pract. 2006, 71, 256-263. [CrossRef] [PubMed]

18. Troxel, W.M.; Buysse, D.J.; Matthews, K.A.; Kip, K.E.; Strollo, P.J.; Hall, M.; Drumheller, O.; Reis, S.E. Sleep symptoms predict the development of the metabolic syndrome. Sleep 2010, 33, 1633-1640. [CrossRef] [PubMed]

19. Tran, B.T.; Jeong, B.Y.; Oh, J.K. The prevalence trend of metabolic syndrome and its components and risk factors in Korean adults: Results from the Korean national health and nutrition examination survey 2008-2013. BMC Public Health 2017, 17, 71. [CrossRef] [PubMed]

20. Lee, W.; Nagubadi, S.; Kryger, M.H.; Mokhlesi, B. Epidemiology of Obstructive Sleep Apnea: A Population-based Perspective. Expert Rev. Respir. Med. 2008, 2, 349-364. [CrossRef] [PubMed]

21. Kim, Y.; Han, B.G. Cohort profile: The Korean genome and epidemiology study (KOGES) consortium. Int. J. Epidemiol. 2017, 46, 1350. [CrossRef] [PubMed]

22. Health Examinees Study Group. The health examinees (HEXA) study: Rationale, study design and baseline characteristics. Asian Pac. J. Cancer Prev. 2015, 16, 1591-1597.

23. Shin, S.; Lee, H.W.; Kim, C.E.; Lim, J.; Lee, J.K.; Lee, S.A.; Kang, D. Egg consumption and risk of metabolic syndrome in Korean adults: Results from the health examinees study. Nutrients 2017, 9, 687. [CrossRef] [PubMed]

24. Grundy, S.M.; Cleeman, J.I.; Daniels, S.R.; Donato, K.A.; Eckel, R.H.; Franklin, B.A.; Gordon, D.J.; Krauss, R.M.; Savage, P.J.; Smith, S.C., Jr.; et al. Diagnosis and management of the metabolic syndrome: An American heart association/national heart, lung, and blood institute scientific statement. Circulation 2005, 112, 2735-2752. [CrossRef] [PubMed]

25. Jordan, A.S.; McEvoy, R.D. Gender differences in sleep apnea: Epidemiology, clinical presentation and pathogenic mechanisms. Sleep Med. Rev. 2003, 7, 377-389. [CrossRef] [PubMed]

26. Bixler, E.O.; Vgontzas, A.N.; Lin, H.M.; Ten Have, T.; Rein, J.; Vela-Bueno, A.; Kales, A. Prevalence of sleep-disordered breathing in women: Effects of gender. Am. J. Respir. Crit. Care Med. 2001, 163, 608-613. [CrossRef] [PubMed]

27. De Sousa, A.G.; Cercato, C.; Mancini, M.C.; Halpern, A. Obesity and obstructive sleep apnea-hypopnea syndrome. Obes. Rev. 2008, 9, 340-354. [CrossRef] [PubMed]

28. Sun, L.; Pan, A.; Yu, Z.; Li, H.; Shi, A.; Yu, D.; Zhang, G.; Zong, G.; Liu, Y.; Lin, X. Snoring, inflammatory markers, adipokines, and metabolic syndrome in apparently healthy Chinese. PLoS ONE 2011, 6, e27515. [CrossRef] [PubMed]

29. Krol, R.C.; Knuth, S.L.; Bartlett, D., Jr. Selective reduction of genioglossal muscle activity by alcohol in normal human subjects. Am. Rev. Respir. Dis. 1984, 129, 247-250. [PubMed]

30. Li, D.; Liu, D.; Wang, X.; He, D. Self-reported habitual snoring and risk of cardiovascular disease and all-cause mortality. Atherosclerosis 2014, 235, 189-195. [CrossRef] [PubMed]

31. Endeshaw, Y.; Rice, T.B.; Schwartz, A.V.; Stone, K.L.; Manini, T.M.; Satterfield, S.; Cummings, S.; Harris, T.; Pahor, M.; Health, A.B.C.S. Snoring, daytime sleepiness, and incident cardiovascular disease in the health, aging, and body composition study. Sleep 2013, 36, 1737-1745. [CrossRef] [PubMed] 
32. Marshall, N.S.; Wong, K.K.; Cullen, S.R.; Knuiman, M.W.; Grunstein, R.R. Snoring is not associated with all-cause mortality, incident cardiovascular disease, or stroke in the Busselton health study. Sleep 2012, 35, 1235-1240. [CrossRef] [PubMed]

33. Lee, S.A.; Amis, T.C.; Byth, K.; Larcos, G.; Kairaitis, K.; Robinson, T.D.; Wheatley, J.R. Heavy snoring as a cause of carotid artery atherosclerosis. Sleep 2008, 31, 1207-1213. [PubMed]

34. Rich, J.; Raviv, A.; Raviv, N.; Brietzke, S.E. An epidemiologic study of snoring and all-cause mortality. Otolaryngol. Head Neck Surg. 2011, 145, 341-346. [CrossRef] [PubMed]

35. Rapoport, D.M. Snoring-obnoxious (but medically innocent) noise or wakeup call for sleep medicine? Sleep Med. Rev. 2014, 18, 451-452. [CrossRef] [PubMed]

36. Fletcher, E.C. Sympathetic over activity in the etiology of hypertension of obstructive sleep apnea. Sleep 2003, 26, 15-19. [CrossRef] [PubMed]

37. Iiyori, N.; Alonso, L.C.; Li, J.; Sanders, M.H.; Garcia-Ocana, A.; O’Doherty, R.M.; Polotsky, V.Y.; O’Donnell, C.P. Intermittent hypoxia causes insulin resistance in lean mice independent of autonomic activity. Am. J. Respir. Crit. Care Med. 2007, 175, 851-857. [CrossRef] [PubMed]

38. Fernandez, A.R.; Rubinos, C.G.; Alonso, A.R.; Cascon, H.J.A.; Palomo, A.B.; Iscar, U.M.; Casan, C.P. Snoring as a determinant factor of oxidative stress in the airway of patients with obstructive sleep apnea. Lung 2016, 194, 469-473. [CrossRef] [PubMed]

39. Shin, C.; Kim, J.; Kim, J.; Lee, S.; Shim, J.; In, K.; Kang, K.; Yoo, S.; Cho, N.; Kimm, K.; et al. Association of habitual snoring with glucose and insulin metabolism in nonobese Korean adult men. Am. J. Respir. Crit. Care Med. 2005, 171, 287-291. [CrossRef] [PubMed]

40. Facco, F.L.; Grobman, W.A.; Kramer, J.; Ho, K.H.; Zee, P.C. Self-reported short sleep duration and frequent snoring in pregnancy: Impact on glucose metabolism. Am. J. Obstet. Gynecol. 2010, 203, 142.e1-142.e5. [CrossRef] [PubMed]

41. Marchesini, G.; Pontiroli, A.; Salvioli, G.; Novi, R.F.; Vitacolonna, E.; Taboga, C.; Ciccarone, A.M.; Grossi, E.; Group, Q.S. Snoring, hypertension and type 2 diabetes in obesity. Protection by physical activity. J Endocrinol. Investig. 2004, 27, 150-157. [CrossRef] [PubMed]

42. Ozol, D.; Carlioglu, A.; Karamanli, H.; Akgedik, R.; Karakurt, F.; Yildirim, Z. Influence of snoring on microalbuminuria in diabetic patients. Sleep Breath. 2011, 15, 295-300. [CrossRef] [PubMed]

43. Joo, S.; Lee, S.; Choi, H.A.; Kim, J.; Kim, E.; Kimm, K.; Kim, J.; Shin, C. Habitual snoring is associated with elevated hemoglobin a1c levels in non-obese middle-aged adults. J. Sleep Res. 2006, 15, 437-444. [CrossRef] [PubMed]

44. Hu, Y.H.; Kuo, S.W.; Wu, D.A. Relationships between hemoglobin and each component of metabolic syndrome: A special focus on elderly without medication. Int. J. Gerontol. 2016, 10, 22-27. [CrossRef] 